\title{
Structural Noise Reduction and Its Effects on Plate Vibrations
}

\author{
R. TROJANOWSKI* AND J. WiCIAK
}

AGH University of Science and Technology, Faculty of Mechanical Engineering and Robotics

Department of Mechanics and Vibroacoustics, al. A. Mickiewicza 30, 30-059 Krakow, Poland

\begin{abstract}
The aim of this work is reduction of structural noise generated by plate and its impact on plates vibrations. For this purpose a one-side clamped aluminum plate with 5 piezo elements attached is used. One of the elements is used for plate excitation, two as vibration sensors, and two as actuators. Structural noise is measured by a microphone connected with SVAN 912 E. Study was divided into three parts: measurements of vibrations and noise generated by excited plate, active vibration control, and structural noise reduction. A significant noise local noise reduction is obtained, although with increase in plate vibrations.
\end{abstract}

PACS: 43.40.Dx, 43.40.-r, 46.40.Ff

\section{Introduction}

In today's world the effects of vibration and noise are becoming quite a severe issue. Both can have a big influence on people's health, and additionally vibrations are usually detrimental to machine performance and sometimes its lifespan. To lower the unwanted effects of noise and/or vibrations passive, active or combinations of both types of methods can be used. In active methods there is a group that uses piezo elements as actuators. Since their introduction in works of Fuller, Dimitradis [1], these methods have progressed. Nowadays there are better materials, computational power of computers allow us to perform real time calculations. Today main issues that need to be dealt with are: optimal actuator placements [2-4], control type [5-7], creation and optimization of controllers [8-10]. There are methods of using piezo elements for passive damping as part of RLC circuits [11], or using acoustic field for non contact vibration control and measurements [12]. Other works deal with noise control by means of using piezo elements either as a sound source $[13,14]$, or combining it with passive solution to improve transmission loss [15].

\section{Description}

This article presents the effects on preliminary studies on structure generated noise. Being a continuation of work presented in [10], it adds structural noise control and measurements to already existing active vibration control (although because of number of microphones used it is rather a local approach). Our object of interest is a one-side clamped aluminum plate. Plate excitation is achieved by vibration signal applied through piezo element (Fig. 1, element W). This excitation is a sinusoidal signal of a given plate resonance frequency from range 100-3000 Hz. Two other piezo elements act as vibration sensors (Fig. 1, elements S1, S2), and another two as actuators (Fig. 1, A1, A2). All elements are ceramic PIC140, and are attached to the plate through a thin layer of epoxy resin.

Vibration signal generation, acquisition and control are handled by a PC with NI 6230 acquisition card and LabVIEW software. For structural noise measurements $1 / 2^{\prime \prime}$ microphone with SVAN 912 E was used. Each time structural noise was measured broadband on linear filter, A filter, and with A filter on specific frequency.

Tests were carried out in anechoic chamber in Laboratory of Technical Acoustics (AGH University of Science and Technology, Kraków, Faculty of Mechanical Engineering and Robotics - Figs. 2, 3).

Piezo elements layout is shown in Fig. 1. Microphone was positioned about $1 \mathrm{~m}$ from the plate in plate's center axis (Fig. 3).

The study is divided into three parts:

1) Part one

Measurements of vibrations and structural noise of excited plate made for each of 11 identified resonance frequencies.

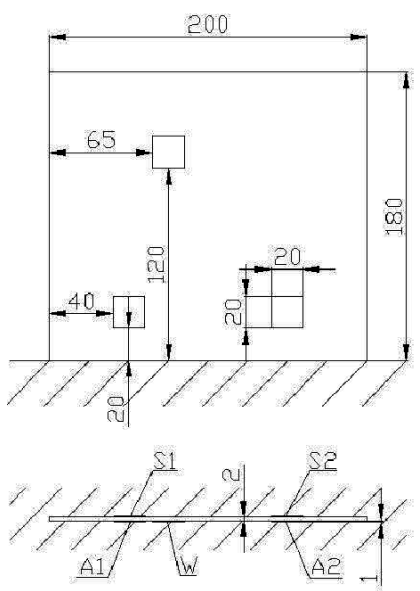

Fig. 1. Distribution of piezo elements.

\footnotetext{
* corresponding author; e-mail:

roman.cz.trojanowski@agh.edu.pl
} 


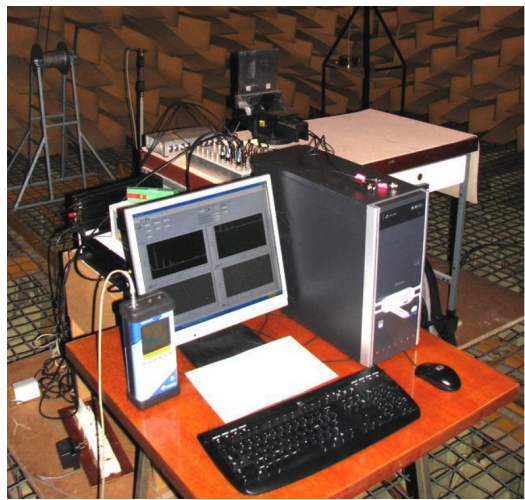

It is worth mentioning that for some frequencies the results are limited because of available voltage amplification means. $\pm 95 \mathrm{~V}$ was the maximum voltage that could be used in study to the actuators. With stronger signals applied to actuators it also would be possible to apply stronger excitation to the plate which would mean better $\mathrm{S} / \mathrm{N}$ ratio.

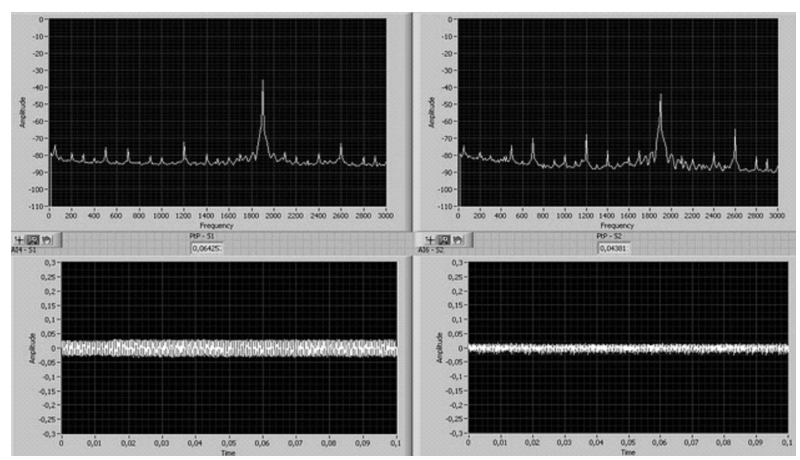

Fig. 4. Vibrations measured for case 2 - active vibration control.

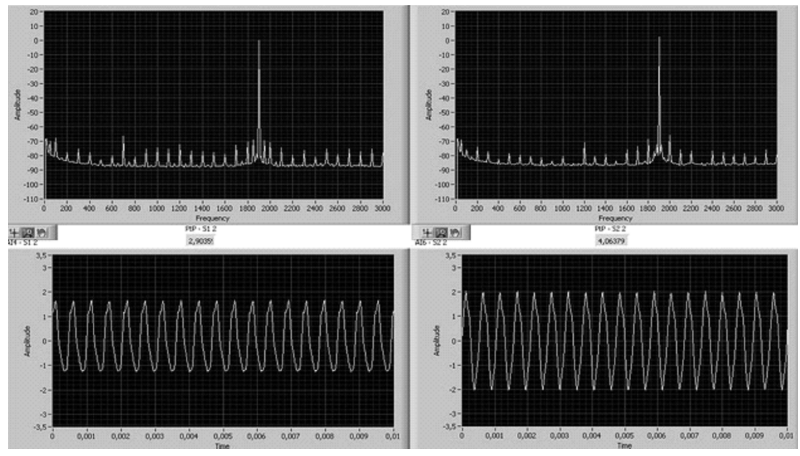

where $x_{i}$ is the sensor voltage signal, $N$ is the number of independent sensors used.

3) Part three

Structural noise reduction with plate vibration measurements. In this part the optimization of voltage applied to actuators (choosing magnitudes and phases) was done manually, because of the lack of connection between the plate's vibrations and noise acquisition tracks. The control cost function was

$$
J=\min x_{i},
$$

where $x_{i}$ is in this case the current broadband sound pressure value with A filter (taken as the most reliable because of cutting low frequency noise disturbances and being less prone to disturbances from measurers movements).

\section{Results}

Results of experiment are shown in Table I. Although the first notable resonance frequency for plate vibrations was $110 \mathrm{~Hz}$, there was no increase in observed noise level. Therefore that frequency is not shown in results.

Fig. 5. Vibrations measured for case 3 - structural noise reduction.

Typical results for the study, where vibrations measured in case of structural noise reduction exceed vibrations measured during vibration control (although not necessarily vibrations without reduction) are shown in Figs. 4 and 5. Also for some frequencies the voltage generated by sensors exceeded acquisition card maximum input. It is assumed that this was because of the change in the distribution of plate's vibration.

When measuring noise with LIN filter (Fig. 6) an average reduction of $8.8 \mathrm{~dB}$ was obtained. For most frequencies that meant reducing the noise to the level of about $50 \mathrm{~dB}$, which as can be seen in Table II was the level of background noise.

Results presented in Fig. 7 look more interesting. When measured on filter A noise generated by plate is reduced significantly. The average reduction observed is about $19.9 \mathrm{~dB}$. Again considering background noise there seem to be two frequencies that probably could be reduced even further. 
TABLE I

Results table: $f$ - given frequency, case: 1 - measurements without vibration reduction, 2 - measurements with vibration reduction, 3 - measurements with noise reduction, $A_{\mathrm{w}}$ - excitation amplitude voltage, $L_{\mathrm{EQ}}-$ noise level measured with LIN filter, $L_{\mathrm{EQA}}$ - noise level measured with A filter, $L_{\mathrm{EQf}}$ - noise level measured for specific frequency, $S_{1}, S_{2}$ - signal amplitudes from sensors, $A_{1}, A_{2}$ - signal amplitudes applied to actuators, $\varphi_{1}, \varphi_{2}$ - signal phases applied to actuators.

\begin{tabular}{|c|c|c|c|c|c|c|c|c|c|c|c|}
\hline $\begin{array}{c}f \\
{[\mathrm{~Hz}]}\end{array}$ & Case & $\begin{array}{l}A_{\mathrm{w}} \\
{[\mathrm{V}]}\end{array}$ & $\begin{array}{l}L_{\mathrm{EQ}} \\
{[\mathrm{dB}]}\end{array}$ & $\begin{array}{c}L_{\mathrm{EQA}} \\
{[\mathrm{dB}]}\end{array}$ & $\begin{array}{c}L_{\mathrm{EQf}} \\
{[\mathrm{dB}]}\end{array}$ & $\begin{array}{c}S_{1} \\
{[\mathrm{~V}]}\end{array}$ & $\begin{array}{c}S_{2} \\
{[\mathrm{~V}]}\end{array}$ & $\begin{array}{c}A_{1} \\
{[\mathrm{~V}]}\end{array}$ & $\begin{array}{l}\varphi_{1} \\
{\left[{ }^{\circ}\right]}\end{array}$ & $\begin{array}{l}A_{2} \\
{[\mathrm{~V}]}\end{array}$ & $\begin{array}{l}\varphi_{2} \\
{\left[^{\circ}\right]} \\
\end{array}$ \\
\hline \multirow{3}{*}{350} & 1 & \multirow{3}{*}{54} & 55.8 & 49.7 & 54.9 & 0.186 & 0.253 & 0 & 0 & 0 & 0 \\
\hline & 2 & & 55.8 & 49.7 & 55.3 & 0.018 & 0.021 & 4 & 213 & 7 & 211 \\
\hline & 3 & & 50.8 & 42.4 & 47.6 & 3.916 & 3.574 & 95 & 12 & 95 & 12 \\
\hline \multirow{3}{*}{780} & 1 & \multirow{3}{*}{54} & 56.4 & 54.8 & 54.0 & 0.749 & 0.742 & 0 & 0 & 0 & 0 \\
\hline & 2 & & 56.9 & 54.9 & 54.5 & 0.041 & 0.042 & 12 & 357 & 17 & 105 \\
\hline & 3 & & 47.5 & 29.7 & 10.9 & 5.160 & 4.680 & 95 & 20 & 95 & 192 \\
\hline \multirow{3}{*}{930} & 1 & \multirow{3}{*}{54} & 52.1 & 51.1 & 49.9 & 1.046 & 1.263 & 0 & 0 & 0 & 0 \\
\hline & 2 & & 51.9 & 48.4 & 47.9 & 0.044 & 0.043 & 16 & 143 & 22 & 334 \\
\hline & 3 & & 48.7 & 25.3 & 17.9 & 4.558 & 2.312 & 95 & 153 & 56 & 323 \\
\hline \multirow{3}{*}{1280} & 1 & \multirow{3}{*}{54} & 68.3 & 68.9 & 69.2 & 0.556 & 0.478 & 0 & 0 & 0 & 0 \\
\hline & 2 & & 68.7 & 69.2 & 69.3 & 0.046 & 0.031 & 10 & 104 & 8 & 103 \\
\hline & 3 & & 63.2 & 63.5 & 63.2 & 5.454 & 5.257 & 95 & 190 & 95 & 187 \\
\hline \multirow{3}{*}{1580} & 1 & \multirow{3}{*}{54} & 65.4 & 66.0 & 64.5 & 1.719 & 1.952 & 0 & 0 & 0 & 0 \\
\hline & 2 & & 60.3 & 61.1 & 59.7 & 0.058 & 0.050 & 17 & 337 & 20 & 337 \\
\hline & 3 & & 44.0 & 26.4 & 15.8 & 3.605 & 1.162 & 60 & 2 & 19 & 0 \\
\hline \multirow{3}{*}{1750} & 1 & \multirow{3}{*}{54} & 57.1 & 57.4 & 57.4 & 2.666 & 1.947 & 0 & 0 & 0 & 0 \\
\hline & 2 & & 58.7 & 59.3 & 59.0 & 0.700 & 0.720 & 59 & 213 & 33 & 34 \\
\hline & 3 & & 49.8 & 41.6 & 35.0 & 3.405 & 1.154 & 42 & 310 & 30 & 8 \\
\hline \multirow{3}{*}{1900} & 1 & \multirow{3}{*}{54} & 58.5 & 59.4 & 58.2 & 0.732 & 1.139 & 0 & 0 & 0 & 0 \\
\hline & 2 & & 56.8 & 57.9 & 55.8 & 0.045 & 0.062 & 4 & 113 & 20 & 165 \\
\hline & 3 & & 47.9 & 41.9 & 27.2 & 4.633 & 2.187 & 92 & 124 & 15 & 154 \\
\hline \multirow{3}{*}{2090} & 1 & \multirow{3}{*}{54} & 66.2 & 67.2 & 65.8 & 1.189 & 0.993 & 0 & 0 & 0 & 0 \\
\hline & 2 & & 65.3 & 66.5 & 65.6 & 0.068 & 0.059 & 23 & 309 & 17 & 285 \\
\hline & 3 & & 53.3 & 33.9 & 27.4 & 4.797 & 2.949 & 94 & 21 & 48 & 7 \\
\hline \multirow{3}{*}{2460} & 1 & \multirow{3}{*}{54} & 76.7 & 77.7 & 76.3 & 4.317 & 1.423 & 0 & 0 & 0 & 0 \\
\hline & 2 & & 76.1 & 77.4 & 75.8 & 0.125 & 0.066 & 37 & 148 & 26 & 315 \\
\hline & 3 & & 55.3 & 54.2 & 52.8 & $>20$ & 15.012 & 95 & 322 & 95 & 9 \\
\hline \multirow{3}{*}{2580} & 1 & \multirow{3}{*}{54} & 60.1 & 61.2 & 59.4 & 5.025 & 4.546 & 0 & 0 & 0 & 0 \\
\hline & 2 & & 54.7 & 56.4 & 54.0 & 0.079 & 0.095 & 47 & 342 & 44 & 159 \\
\hline & 3 & & 51.5 & 33.1 & 28.5 & 3.453 & 2.00 & 95 & 5 & 25 & 180 \\
\hline \multirow{3}{*}{2850} & 1 & \multirow{3}{*}{54} & 49.8 & 49.4 & 49.6 & 0.942 & 0.858 & 0 & 0 & 0 & 0 \\
\hline & 2 & & 53.6 & 54.5 & 53.8 & 0.058 & 0.048 & 12 & 302 & 13 & 111 \\
\hline & 3 & & 47.9 & 32.3 & 24.6 & 1.476 & 1.021 & 10 & 89 & 0 & 0 \\
\hline
\end{tabular}

With measurements for specific frequency (Fig. 8) it can be seen that for some of them noise was almost completely eliminated (like $780 \mathrm{~Hz}$ ). Average reduction is further increased to $25.7 \mathrm{~dB}$. It should be noted, however, that even the slightest change in control signals (like changing signal's phase $1^{\circ}-3^{\circ}$ ) could increase measured noise even $15 \mathrm{~dB}$.

In Figs. 6-8 it can be seen that for some frequencies $(350 \mathrm{~Hz}, 1280 \mathrm{~Hz}$, and $2460 \mathrm{~Hz})$ noise levels are almost unchanging. Although some reduction can be observed (respectively $7 \mathrm{~dB}, 6 \mathrm{~dB}$, and $23 \mathrm{~dB}$ ), measured noise level is still much higher than in other cases. This could be probably explained by two things: position of microphone and position of actuators. Because there is only one stationary position of microphone we can only talk about local noise reduction. It is also possible that this specific position of actuators is not very effective for these frequencies. 


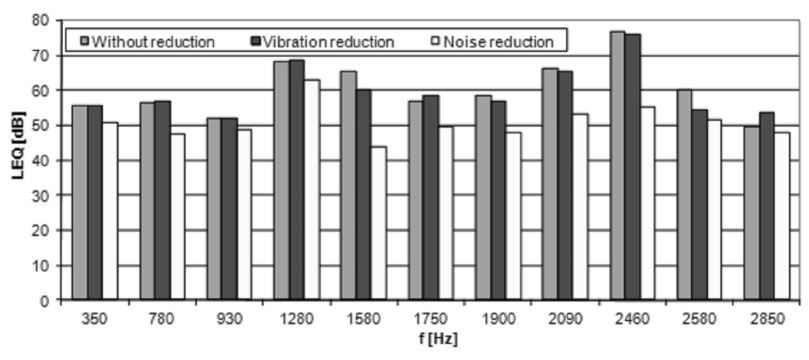

Fig. 6. Noise measured with LIN filter.

TABLE II

Background information: meas. - number of measurement, $t$ - air temperature, humidity - air humidity, $p$ - air pressure, $L_{\mathrm{EQ}}$ - background noise level measured with LIN filter, $L_{\mathrm{EQA}}$ - background noise level measured with A filter.

\begin{tabular}{c|c|c|c|c|c}
\hline \hline Meas. & $\begin{array}{c}t \\
{\left[{ }^{\circ} \mathrm{C}\right]}\end{array}$ & $\begin{array}{c}\text { humidity } \\
{[\%]}\end{array}$ & $\begin{array}{c}p \\
{[\mathrm{~Pa}]}\end{array}$ & $\begin{array}{c}L_{\mathrm{EQ}} \\
{[\mathrm{dB}]}\end{array}$ & $\begin{array}{c}L_{\mathrm{EQA}} \\
{[\mathrm{dB}]}\end{array}$ \\
\hline 1 & 20 & 60 & 98000 & 49.7 & 24.8 \\
2 & 20 & 60 & 98600 & 49.2 & 24.7 \\
3 & 20 & 55 & 98500 & 51.5 & 23.8
\end{tabular}

\section{Conclusions}

Presented work shows that piezo elements can be successfully used in structure noise reduction. Results indicate that it was possible to obtain significant reduction, but because of the number of microphones used, we can only talk about local reduction (local zones of quiet). Therefore even if global reduction is not possible, it can be assumed that it is possible to create almost any desired local zone of quiet. Also with completion of control system it should be possible to shift those zones as desired within a certain range, limited by frequencies and actuators placement.

Obtained results seem to confirm literature that noise reduction does not go in pair with vibration reduction. While the vibration reduction did not have almost any effect on noise generated by the plate, noise reduction greatly amplified vibration (up to the point of going above DAQ card range). It should be considered that there was only one microphone and two vibration sensors. Therefore further measurements with additional sensors should be conducted.

A possible improvement to vibration and structural noise control could be a piezo elements matrix, where depending on plate's vibration distribution corresponding elements would be used.

\section{Acknowledgments}

This study is a part of the research project $\mathrm{N}$ N504078038 supported by the Ministry of Science and Higher Education, Poland.

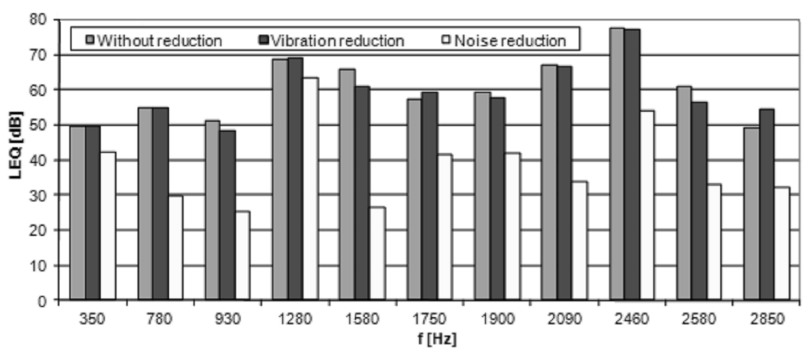

Fig. 7. Noise measured with A filter.

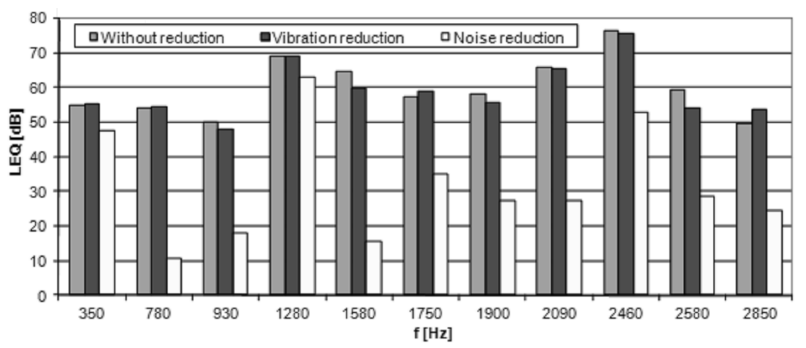

Fig. 8. Noise measured for specific frequency.

\section{References}

[1] E. Dimitradis, C. Fuller, C. Rogers, J. Vibrat. Acoust. 113, 100 (1991).

[2] A. Brański, G. Lipiński, Acta Phys. Pol. A 119, 936 (2011).

[3] A. Brański, M. Borkowski, S. Szela, Acta Phys. Pol. A 118, 17 (2010).

[4] J.D. Sprofera, R.H. Cabell, G.P. Gibbs, R.L. Clark, J. Acoust. Soc. Am. 122, 271 (2007).

[5] C.R. Fuller, S.J. Elliott, P.A. Nelson, Active Control of Vibration, Academic Press, 1996.

[6] C.H. Hansen, S.D. Snyder, Active Control of Noise and Vibration, E\&FN Spon, 1997.

[7] P. Gardonio, S.J. Elliott, J. Acoust. Soc. Am. 117, 2046 (2005).

[8] P. Gardonio, Sensor-Actuator Transducers for Smart Panels, ACTIVE, Australia 2006.

[9] K. Pietrusiewicz, Control Eng. Poland 5, 20 (2007).

[10] R. Trojanowski, J. Wiciak, Acta Phys. Pol. A 118, 168 (2010).

[11] M.S. Kozień, B. Kołtowski, Acta Phys. Pol. A 119, 1005 (2011).

[12] A. Brański, S. Szela, Acta Phys. Pol. A 119, 942 (2011).

[13] M.M. Zhang, L. Cheng, Y. Zhou, J. Acoust. Soc. Am. 126, 36 (2009).

[14] N. Tanaka, M. Tanaka, J. Acoust. Soc. Am. 127, 3526 (2010).

[15] A. Kundu, A. Berry, J. Acoust. Soc. Am. 129, 726 (2011). 